\title{
El cómputo ubicuo y su importancia para la construcción del internet de las cosas y el big data
}

\author{
Georgina Araceli TORRES VARGAS \\ gtorres.ara@gmail.com \\ Raquel ARIAS DURÁ ${ }^{1}$ \\ raqueldura12@gmail.com \\ Universidad Nacional Autónoma de México. Instituto de Investigaciones \\ Bibliotecológicas y de la Información
}

Recibido: Septiembre 2014

Aceptado: Noviembre 2014

Resumen: Se parte de la idea de cómputo ubicuo de Mark Weiser, para plantear en qué medida el logro de propuestas como el internet de las cosas y big data dependen de las bases dadas por Weiser. Para ello se muestran las características que teóricamente tienen tanto el big data como el internet de las cosas. El objetivo principal del artículo es analizar qué adelantos se requieren para el logro de estas dos concepciones tecnológicas y de manejo de la información.

Palabras clave: Big data; Computación ubicua; Internet de las cosas.

The ubiquitous computing and its importance for the construction of the internet of things and big data

\begin{abstract}
It starts from the idea of ubiquitous computing Mark Weiser, the extent to raise the achievement of proposals such as the internet of things and big data depend on the bases given by Weiser. Showing what are the characteristics of the Big data and the Internet of things. The main objective of the paper is to analyze what progress is required to achieve these two technological concepts and information management.
\end{abstract}

Keywords: Big data; Internet of things Ubiquitous computing.

\section{INTRODUCCIÓN}

El alto desarrollo e impacto de las tecnologías de la información y de la comunicación (TIC) en la vida cotidiana y en diversas áreas del conocimiento, ha

\footnotetext{
${ }^{1}$ Investigación realizada gracias al Programa de Becas Posdoctorales en la UNAM 
creado una necesidad por analizarlas y conocer sus repercusiones. Tal es el caso de la bibliotecología y de los estudios de la información, al ser disciplinas que se ven directamente alteradas por los nuevos usos de la información digital.

Si bien a primera vista pareciera que las TIC llegaron de manera intempestiva, éstas se fueron desarrollando de forma paulatina gracias a los aportes de diferentes estudiosos que hoy se califican de visionarios, pero que más que adelantarse a su época, sentaron las bases para construir un futuro tecnológico más amigable.

Tal es el caso del aporte de Mark Weiser a través de su propuesta de cómputo ubicuo, aspecto que en la actualidad requiere retomarse para comprender muchos de los elementos que están presentes en el manejo de información digital, como el big data y el internet de las cosas.

\section{CÓMPUTO UBICUO}

A finales de la década de 1980 Mark Weiser desarrolló un programa para Xerox PARC, al que llamó Ubicomp (computación ubicua) ${ }^{2}$

Para Weiser la computación ubicua constituiría la tercera ola de la computación; su idea central descansa en el diseño de un modelo diferente del que en ese momento existía y que se apoyaba en el uso del ordenador personal tradicional. Su desafío era crear un entorno tecnológico en donde dispositivos de diferentes tamaños y funcionalidades, pudieran conectarse y usarse en conjunto para manejar información, de forma tal que el hombre operara con mayor facilidad sus actividades del mundo cotidiano ${ }^{3}$ La computación ubicua es en opinión de Weiser, lo contrario de la realidad virtual pues mientras la realidad virtual pone a las personas en un mundo generado por computadora, la computación ubicua hace que la tecnología ayude a la gente a vivir en el mundo real ${ }^{4}$

Con esta meta, se hizo realidad el sistema Ubicomp en 1992, producto del seguimiento y mejora de programas como: "the board, the pad, the tab, the badge"... ${ }^{5}$-que es como los nombró Weiser- desarrollados desde 1988 y dirigidos hacia la constitución de una infraestructura que permitiera identificar cada dispositivo, su localización, uso, conectividad y usuario. Dos de sus prototipos se describen a continuación. ${ }^{6}$

\footnotetext{
${ }^{2}$ Want, 2000: 8 .

${ }^{3}$ Weiser, 1999: 693

${ }^{4}$ Weiser, 1999: 693

${ }^{5}$ Ibid: 694.

${ }^{6}$ Weiser, 1993: 77.
} 


\section{$T a b$}

El ParcTab lo describió Weiser como una puerta de entrada a la información. Contaba con una pantalla sensible a la presión (táctil) y tres botones debajo. $\mathrm{Su}$ principal problema fue el alto consumo de energía.

\section{Pad}

Dispositivo portátil que podía usarse como bloc de notas. Se contó con seis versiones del Pad; su principal problema fue la falta de un sistema operativo adecuado.

El Ubiquitous Computing project que Weiser desarrolló en Xerox PARC, integraba elementos de la informática, la ingeniería y las ciencias sociales. Su noción de computación ubicua, la describe a través de dos tiempos, tal y como se observa en la siguiente tabla:

\begin{tabular}{|c|c|}
\hline Computación ubicua \#1 & Computación ubicua \#2 \\
\hline $\begin{array}{l}\text { La gente vive a través de sus prácticas, por } \\
\text { lo que su conocimiento tácito es } \\
\text { importante. El primer reto de la } \\
\text { computación ubicua es activar el mundo; } \\
\text { proporcionar cientos de dispositivos } \\
\text { informáticos inalámbricos } \\
\text { Por persona, por oficina, a todas las escalas. } \\
\text { Este trabajo requiere de sistemas } \\
\text { operativos, interfaces, redes inalámbricas, } \\
\text { pantallas y muchas otras cosas. Esto es a lo } \\
\text { que llamamos "computación ubicua" } \\
\text { porque no reside en un dispositivo, pues } \\
\text { está en todas partes. }\end{array}$ & $\begin{array}{l}\text { En esta fase la mayoría de los diseños de } \\
\text { interfaces, y del diseño por ordenador, ha } \\
\text { sido encaminada hacia el desarrollo de un } \\
\text { equipo tan emocionante, tan maravilloso, } \\
\text { tan interesante, que desearemos no estar sin } \\
\text { él. El objetivo es hacer que un equipo se } \\
\text { arraigue de manera natural y que lo } \\
\text { utilicemos sin siquiera pensar en ello } \\
\text { (también he llamado a esta noción } \\
\text { "computación ubicua", y tiene sus orígenes } \\
\text { en el post-modernismo.) Creo que en los } \\
\text { próximos veinte años, este segundo } \\
\text { escenario dominará pero no será fácil llegar } \\
\text { allí. }\end{array}$ \\
\hline
\end{tabular}

Tomada de: Weiser, Mark Ubiquitous computing. ${ }^{\text {? }}$

Weiser da las bases para algunos avances recientes como el internet de las cosas y big data, aunque puede aseverarse que todavía no se alcanza el grado de desarrollo suficiente para asegurar que tenemos enfrente al cómputo ubicuo, analicemos por qué.

${ }^{7}$ Cfr. Weiser, 1993: 76. 


\section{INTERNET DE LAS COSAS}

El internet de las cosas (IdC) es un término propuesto por Kevin Ashton en 1999 durante una presentación en la que argumentó que mediante la asociación de los objetos físicos con etiquetas RFID $^{8}$, se podría dar identidad a cada objeto para generar datos sobre tales cosas. Lo novedoso de esta cuestión es que hasta el momento la información disponible en internet se había producido casi exclusivamente por individuos o por sistemas computarizados (por ejemplo, información de vuelos), pero no por las cosas físicas.

El internet de las cosas es el entorno tecnológico en el que los objetos cotidianos estarán conectados a internet y serán capaces de recibir, generar y enviar información. Se estima que para el año 2015 habrá más de 200 mil millones de dispositivos conectados a internet; cuatro veces más que en 2010. En unos cuantos años, internet de las cosas será una estructura compleja, en donde se espera que haya miles de millones de nodos capaces de medir cualquier cosa medible, así como de extraer y comunicar cualquier forma de información. Esta información se podrá utilizar para controlar casi todos los aspectos de la vida en el mundo real. ${ }^{9}$

¿Cómo funcionarán estas cosas conectadas al internet? Juan Ignacio Vázquez ${ }^{10}$ da un ejemplo ilustrativo:

Una silla inteligente parece una silla común, pero el respaldo y el asiento cuentan con un conjunto de pequeños sensores que registran continuamente la postura del usuario. Un módulo inalámbrico envía los datos de la postura de la persona a un conjunto de servidores, donde se almacenan los datos y se analizan los patrones que reflejen si se tiene una buena postura, si se pasa demasiado tiempo en la misma posición, o no se tiene suficientes lapsos de descanso. Esta información puede ayudar al usuario de la silla inteligente a mejorar su postura y aliviar problemas de espalda o bien prevenir esos problemas. Algunas sillas inteligentes vibran cuando detectan alguna postura que no es adecuada y hace que el usuario aprenda y adoptar una buena postura.

Para el usuario uno de los grandes problemas será el control de las cosas a través de una sola plataforma, ya que cada empresa ofrecerá la propia y será complicado para mantener el registro y seguimientos de cada dispositivo.

${ }^{8}$ La tecnología RFID (Radio Frequency IDentification), cuyo objetivo es el de identificar objetos de una forma rápida, con poca transmisión de información y en un entorno cercano. Las etiquetas RFID sirven para transmitir información codificada aantenas especiales. (Cfr. ALEXANDRES FERNÁNDEZ, Sadot, 2006).

\footnotetext{
${ }^{9}$ González, 2013.

${ }^{10}$ Vázquez, 2014.
} 


\section{BIG DATA}

A diario se crean inmensas cantidades de datos nuevos que ya no pueden ser procesados por las herramientas tradicionales; como datos de redes sociales, registros de web, datos de sensores y no estructurados. Estos datos procesados y almacenados por las instituciones rebasan las infraestructuras arquitectónicas del sector del almacenamiento actual.

Se estima que en el mundo anualmente se produce una media de poco menos de un terabyte ${ }^{11}$ por persona y que en colectivo, se producen en el mundo cerca de cinco zettabytes $^{12}$ de datos por año. ${ }^{13}$

Los grandes volúmenes de datos superan las infraestructuras de almacenamiento actuales en tres grandes áreas.

- Complejidad. La información ahora está relacionada y está formada por múltiples datos que no son solo texto y números, incluyen eventos en tiempo real y de infraestructura compartida. Cada vez resulta más insuficiente y elaborado aplicar los algoritmos normales para la búsqueda, almacenamiento y categorización.

- Velocidad. El vídeo de alta definición o la retransmisión multimedia a través de dispositivos reproductores de Internet, entre otros, tienen altas tasas de consumo. Los negocios tienen que seguir el ritmo de los flujos de datos para que la información sea útil y también el ritmo de las tasas de incorporación de datos para ofrecer resultados más rápidos, o en el caso militar o médico, para salvar vidas.

- Volumen. Todos estos datos deben ser almacenados en una ubicación segura y siempre disponible. Con volúmenes de datos tan altos, los equipos técnicos tienen que decidir si se desechan datos cada semana o si se almacenan por más tiempo, pero sin aumentar la complejidad operativa, y esto puede provocar fallos en la infraestructura de manera continua.

Como puede verse, el big data es un término aplicado a conjuntos de datos que superan la capacidad del software habitual para ser capturados, gestionados y procesados en un tiempo razonable. Es el tratamiento informatizado de grandes cantidades de información, la definición no ha variado mucho con el paso del tiempo ya que los sistemas informáticos son cada vez más potentes y pueden almacenar y procesar cada vez más datos de lo que antes se podía.

${ }^{11}$ Para darnos una idea de lo que representa un terabyte, puede decirse que equivale aproximadamente a 300 horas de vídeo o 3,6 millones de fotografías digitales.

${ }^{12}$ Un zettyabyte equivale a 1000000000000000000000 bytes

${ }^{13}$ Aiden, 2013: 11 
Este conjunto de datos se descubre como una fuente inexplorada fruto de esta nueva forma de vida en la que vivimos gracias a las redes sociales y del inmenso número de dispositivos que nos mantienen conectados. El volumen, la velocidad y la variedad de estos datos muestran que las empresas que no están equipadas para analizarlos y extraer su valor empresarial potencial, presentan retos significativos $\mathrm{y}$ un gran potencial para ayudar a estas empresas a competir y avanzar.

Tipos de datos

1.-Web and Social Media: Incluye contenido web e información que es obtenida de las redes sociales como Facebook, Twitter, LinkedIn, etc, blogs.

2.-Machine-to-Machine (M2M): M2M se refiere a las tecnologías que permiten conectarse a otros dispositivos. M2M utiliza dispositivos como sensores o medidores que capturan algún evento en particular (velocidad, temperatura, presión, variables meteorológicas, variables químicas como la salinidad, etc.) los cuales transmiten a través de redes alámbricas, inalámbricas o híbridas a otras aplicaciones que traducen estos eventos en información significativa.

3.-Big Transaction Data: Incluye registros de facturación, en telecomunicaciones registros detallados de las llamadas (CDR), etc. Estos datos transaccionales están disponibles en formatos tanto semiestructurados como no estructurados.

4.-Biometrics: Información biométrica en la que se incluye huellas digitales, escaneo de la retina, reconocimiento facial, genética, etc. En el área de seguridad e inteligencia, los datos biométricos han sido información importante para las agencias de investigación.

5.-Human Generated: Las personas generamos diversas cantidades de datos como la información que guarda un call center al establecer una llamada telefónica, notas de voz, correos electrónicos, documentos electrónicos, estudios médicos, etc.

Las tecnologías del big data no solo deben ayudar a recopilar grandes cantidades de datos, sino que además deben permitir entenderlos y aprovechar su valor para optimizar la toma de decisiones.

De esta forma, autores como Boyd define al Big Data como como un fenómeno cultural, tecnológico y académico, que se apoya en la interacción entre los siguientes elementos:

(1) Tecnología: la maximización de la potencia de cálculo y la precisión algorítmica para

recopilar, analizar, enlazar y comparar conjuntos de datos grandes.

(2) Análisis: sobre la base de grandes conjuntos de datos para identificar patrones de demandas sociales, económicas, técnicas y legales. 
Hay una creencia casi generalizada de que los grandes conjuntos de datos ofrecen una forma superior de la inteligencia y el conocimiento que pueden generar ideas que antes eran imposibles. Pero el Big Data no es un escenario en donde los números hablen por sí mismos.

Por otra parte en el IdC a través de cada dispositivo conectado se obtendría infinidad de datos, por lo que su relación con el Big Data es directa. Esta relación puede notarse muy bien en el ejemplo que se daba anteriormente sobre la silla inteligente, puesto que este objeto se estará supervisando durante el tiempo que se use, lo que arrojará diferentes datos y así además del usuario, el fabricante podrá hacer uso de mucha información para tomar decisiones sobre su producto.

En otro escenario, el Big Data se ve como una herramienta de gran alcance para hacer frente a diversos males que aquejan actualmente a la sociedad, por ejemplo al ofrecer datos que permitan conocer la investigación sobre el cáncer $u$ otras enfermedades el cambio climático, entre muchos otros aspectos. Sin embargo también se ve al big data como una manifestación preocupante, porque permitiría la invasión de la privacidad y la consecuente disminución de las libertades civiles. Al igual que con otros fenómenos socio-técnicos, las percepciones de esperanza y temor se entrecruzan ${ }^{14}$

\section{TECNOLOGÍAS DERIVADAS DEL CÓMPUTO UBICUO QUE AYUDARÍAN A LOGRAR EL INTERNET DE LAS COSAS Y EL BIG DATA}

Como se pudo observar, tanto el IdC como el Big Data harán uso de diversas tecnologías que derivan del cómputo ubicuo, puesto que son un conjunto de dispositivos que en su conjunto manejan datos para que facilitar al individuo sus actividades de la vida diaria.

Si bien hay adelantos en la construcción de una infraestructura de cómputo ubicuo, todavía no se puede hablar de su consolidación ya que muchas de las tecnologías necesitan de más desarrollo. Sin embargo hay asomos de avances y por consiguiente es importante mencionarlos a continuación.

Redes inalámbricas de área corporal (Tecnología WBAN Wireless Body Area Network)

Son redes de comunicación inalámbrica entre dispositivos utilizados sobre el cuerpo humano, por ejemplo micrófonos, auriculares, sensores, etc. Estas redes pueden estar formadas por sensores de baja potencia implantados en el cuerpo que controlan los parámetros vitales del cuerpo y sus movimientos. Estos dispositivos

${ }^{14}$ Boyd, 2011. 
transmiten datos desde el cuerpo a un punto base como puede ser un hospital o clínica, en tiempo real a través de tecnologías inalámbricas. Estas redes surgen con el objetivo primordial de la asistencia médica y la vigilancia continua de los parámetros vitales de los pacientes que padecen enfermedades crónicas y necesitan ser monitoreados las veinticuatro horas del día.

A pesar de usarse esta tecnología WBAN primordialmente en el campo médico, ya se está empezando a aplicar en otros sectores como son los deportes, la electrónica, la seguridad o para aplicaciones militares.

Identificación de radio frecuencia (Radio-frequency identification o RFID)

Puesto que el internet de las cosas depende de ciertos desarrollos como la tecnología RFID, es importante mencionar en qué medida está avanzando.

La tecnología RFID es utilizada para la identificación de un elemento (similar a un número de serie único); gracias a dispositivos pequeños, como una pegatina o una etiqueta especial que se encuentra incorporada al producto y emite ondas de radio. Estos dispositivos leen, capturan y transmiten la información, pueden seguir su ruta de movimiento y hasta calcular las distancias. Una de las ventajas del uso de radiofrecuencia es que no se necesita una visión directa entre emisor y receptor. Estos dispositivos fueron diseñados con antenas para poder recibir y responder las peticiones por radiofrecuencia.

Estas tecnologías permiten la lectura de etiquetas incluso si éstas no se encuentran en una línea visual directa y se agrupan dentro de las denominadas Auto ID (identificación automática).

La tecnología de radio identificación de frecuencias, está formada por tres elementos básicos:

- Un tag, o microchip

- Un lector RFID

- Software

Esta tecnología de radio identificación de frecuencias va en evolución y en el futuro se piensa que permitirá identificar puestos de salud, administrar y controlar historias clínicas y en general automatizar los recursos y procesos hospitalarios, ${ }^{15}$ creando así sistemas de información hospitalarios que ahorren tiempos de espera, errores y que garanticen una mejor atención médica al paciente.

El tag podrá ser colocado en la muñeca del paciente y programado con la historia clínica del mismo, a fin de que las enfermeras y médicos pueden acceder a los datos personales a través de un lector. Esto garantizará mayor rapidez para tener a la mano

${ }^{15}$ Escobar de Lima, 2007. 
los diagnósticos y observaciones sobre la salud del paciente, además de evitar errores por ejemplo en la manipulación y suministro de medicina. ${ }^{16}$

\section{Cosas (u objetos) empáticas (cos)}

El IdC se habrá de reconfigurar a partir de las tecnologías que se vayan desarrollando. Por ejemplo, se espera la aparición de cosas empáticas (empathic things). Vista en tres etapas, la tecnología de la información y la comunicación ha transitado por una etapa personal, una social y pronto estará en una etapa de cosas empáticas, en donde la tecnología será más íntima, centrada en el hombre y ubicua. ${ }^{17}$ Estas cosas, estarán insertas o colocadas en alguna parte del cuerpo humano, a fin de recabar datos y realizar una aplicación específica.

Las cosas empáticas no funcionarán por sí solas. Se requiere de una convergencia de tecnología social, móvil, de procesos y de nube, para que las cosas empáticas puedan ofrecer servicios.

La tecnología de cosas empáticas se clasifican en varios grupos, algunos de los cuales se mencionan a continuación: ${ }^{18}$

\section{Ingeribles}

Por ejemplo, Motorola junto con Proteus Digital Health desarrolla una píldora que monitorea cada cierto tiempo la acidez gástrica y da un registro de los datos. Lo mismo puede hacer con cuestiones cardiacas.

2. Portables

Esto ya es visible en algunos de los gadgets (como las pulseras inteligentes), que monitorizan el rendimiento en carreras o en alguna otra actividad física. En esta categoría se incluyen las Google glass.

3. Implantables

En forma de chip, e implantada debajo de la piel, esta tecnología puede arrojar datos médicos del paciente y comunicarlos mediante red inalámbrica o por bluetooth.

Con cada cosa conectada se obtendría infinidad de datos; en el caso del ejemplo de la silla inteligente, este objeto se está supervisando todo el tiempo el uso que se le da y el fabricante puede hacer uso de mucha información para tomar decisiones sobre su producto

Para el usuario uno de los grandes problemas será el control de las cosas a través de una sola plataforma, ya que cada empresa ofrecerá la propia y será complicado para el usuario mantener el registro y seguimientos de cada dispositivo.

${ }^{16}$ Ibidem.

${ }^{17}$ Empathic things. Intimate computing from wearables to biohacking, 2014.

${ }^{18}$ Ibid. 


\section{Redes de sensores}

Los sistemas de sensores se caracterizan por registrar grandes cantidades de datos que son enviados a la nube, donde son relacionados con otros conjuntos de datos y procesados, en tiempo real, para dar lugar a un conocimiento que se distribuye a través de la red.

En nuestro entorno podemos encontrar numerosas redes de sensores, con finalidades tan diversas como mejorar el rendimiento deportivo, prever riesgos como tsunamis, terremotos, erupciones volcánicas y excesos de radiación, o mejorar la seguridad del tráfico.

\section{Seguridad informática}

La seguridad informática está concebida para proteger los activos informáticos, entre los que se encuentran; la infraestructura computacional, los usuarios y la información.

Se puede definir como el área de la informática que se centra en la protección de la infraestructura computacional y todo lo relacionado con esta y sobretodo la información contenida. Existen una serie de estándares, herramientas y leyes concebidas para minimizar los posibles riesgos a la infraestructura o a la información.

\section{Sistemas distribuidos}

Son colecciones de computadoras conectadas entre sí por una red de comunicaciones y están separadas físicamente; el programador percibe el conjunto como un sistema único donde se puede acceder a los componentes remotos de la misma manera en que se accedería a los locales pero cada máquina posee sus componentes de hardware y software. El tamaño de estos sistemas es muy variado, desde decenas a millones de host (escalabilidad) y deben ser muy confiables y seguros ya que al tratarse de una red conectada, si falla uno de los componentes, otro debe ser capaz de reemplazar sus funciones (tolerancia a fallos).

\section{Computación móvil}

Las redes móviles utilizan servicios telefónicos y servicios públicos para transmitir y recibir señales de forma inalámbrica. Para que esto funcione se utiliza: Comunicación de paquetes vía radio, redes celulares y estaciones de satélite. Es una serie de artefactos y equipos portátiles, que hacen uso de la computación para lograr su funcionamiento, como pueden ser las computadoras portátiles, los teléfonos celulares, los cuadernos de notas computarizados, etc.

\section{Computación en la nube}

La computación en la nube permite ofrecer servicios de computación a través de Internet. En este tipo de computación todo lo que puede brindar un sistema informático se ofrece como servicio, de modo que los usuarios puedan acceder a 
los servicios disponibles en la nube de Internet sin conocimientos avanzados en la gestión de los recursos que usan.

Es un modelo en el que la información se almacena de manera permanente en servidores de Internet y se envía al usuario; ya sea a sus equipos de escritorio, centros de ocio, laptops, etc.

La computación en la nube está formada por servidores que desde Internet se encargan de atender las peticiones en cualquier momento. Se puede tener acceso a su información o servicio, mediante una conexión a internet desde cualquier dispositivo móvil o fijo ubicado en cualquier lugar, de esta forma se reducen costes y se garantiza un mejor tiempo de actividad.

\section{Smart Grid}

Una limitante para el IdC es la carga de energía eléctrica para cada aparato. El reto es hacer que la carga dure mucho más tiempo para que el individuo pueda hacer uso de sus dispositivos sin preocuparse durante sus actividades diarias por conectarlos a la corriente. Vázquez da también en este caso un ejemplo. Se imagina cuán complicado sería tener que cargar la batería de los zapatos inteligentes o poner la sombrilla en modo de espera. ${ }^{19}$

La alternativa para solucionar este problema es el desarrollo de tecnología de smart grid, que se concibe como una red de comunicación de datos, que está soportada sobre un software de administración de energía flexible y transparente, para utilizar eficientemente la energía. ${ }^{20}$

\section{Ejemplos de adelantos recientes.}

Radio system for long-range high-speed wireless communication ${ }^{21}$ (Sistema de radio de largo alcance de comunicación inalámbrica de alta velocidad)

En la patente de este invento, se menciona que el rápido desarrollo de las fibras ópticas, que permiten la transmisión a través de largas distancias y en anchos de banda superiores, ha revolucionado la industria de las telecomunicaciones y ha jugado un papel importante en el advenimiento de la era de la información. Sin embargo, hay limitaciones en la aplicación de las fibras ópticas, debido a que no es rentable extender el alcance de la fibra óptica a zonas escasamente pobladas, como las zonas rurales o hacia áreas difíciles de alcanzar.

Por otro lado, los dispositivos y sistemas de comunicación de radio inalámbricas proporcionan una transmisión de datos a alta velocidad a través de una interfaz de aire, por lo que es una tecnología atractiva para proporcionar conexiones de red a las áreas que aún no son alcanzados por fibras o cables. Sin

\footnotetext{
${ }^{19}$ Vázquez, 2014.

${ }^{20}$ Gungor, $2013: 29$.

${ }^{21}$ Schulz, 2014.
} 
embargo, las tecnologías inalámbricas disponibles actualmente para largo alcance, como las conexiones punto a punto se encuentran con muchos problemas, entre ellas la mala calidad de la señal. Esta invención pretende ser una alternativa para dar solución a este tipo de problemas.

- Mobile application for monitoring and controlling devices ${ }^{22}$ (Aplicación móvil para dispositivos de vigilancia y control)

En la patente de esta tecnología se menciona que las tecnologías de automatización del hogar a menudo se implementan utilizando dispositivos de control y monitores especialmente diseñados que se comunican entre sí mediante un protocolo de comunicación dedicado. Debido a que este protocolo de comunicación entre dispositivos es de propiedad de las empresas, los dueños de casa tienen problemas para personalizar el sistema e incluir diferentes dispositivos de otros proveedores. Por ejemplo, en un sistema de vigilancia de casa, el controlador del sistema de vigilancia está conectado a menudo a varios sensores y / o cámaras diseñadas y fabricadas por el mismo proveedor.

Para implementar el control centralizado, se requiere que los aparatos sean desarrollados por el mismo fabricante y si el dueño de casa también quiere instalar un sistema de riego automatizado, puede que tenga que comprar e instalar un controlador fabricado por un proveedor diferente que el sistema, lo que le impide controlar de forma centralizada.

Para empeorar las cosas, si un usuario desea controlar los sistemas de automatización a través de un ordenador, el usuario necesita interactuar a través de una interfaz de usuario diferente para cada sistema automatizado y si un propietario desea monitorizar los aparatos de un sistema, el dueño de la casa debe utilizar el software proporcionado por el mismo fabricante de los aparatos.

Esta tecnología ofrece una aplicación de monitoreo que se puede ejecutar en un dispositivo móvil, tableta, PC u otro dispositivo portátil, y facilita el control de sensores y la navegación a través de los datos, ya sea directamente o a través de un servicio de gestión de sensor. Un usuario puede controlar una variedad de sensores, tales como un sensor de movimiento, un sensor de temperatura, un sensor de puerta, un sensor eléctrico.

${ }^{22}$ Bauer, 2014. 


\section{CONCLUSIONES}

A partir de lo enunciado se puede concluir lo siguiente:

El Internet de las Cosas y el Big data son dos conceptos que guardan una estrecha relación con el cómputo ubicuo, idea desarrollada por Mark Weiser a finales de la década de 1980 .

El cómputo ubicuo se refiere a un contexto tecnológico en donde dispositivos e infraestructura de redes entre otras cuestiones, hagan más fácil la vida de los seres humanos al proporcionarle datos e información que arrojen sus actividades. En este momento no se puede hablar de un logro del cómputo ubicuo, pero hay propuestas que permiten vislumbrar más nítidamente sus alcances, como pueden ser la Internet de las cosas y Big Data.

El concepto de Internet de las cosas engloba la idea de un mundo en el que todos los objetos cotidianos que nos rodeen tengan una relación digital; que exista una capa de conectividad digital para objetos. Cualquier objeto imaginable es susceptible de ser conectado a la Red, lo que supone enormes avances a lo largo y ancho de las industrias, pero sobretodo estas tecnologías se centran en la domótica, la medicina, la industria farmacéutica y la logística.

El IdC se refiere a las diversas tecnologías que pueden conseguir conectar cualquier objeto a la nube. Pero para que esto se produzca se necesita una tecnología que evolucione y cambios en el paradigma de lo cotidiano. Cuantos más objetos estén enlazados gracias a sensores o etiquetas RFID, nuestro mundo estará más interconectado y estos nuevos dispositivos generarán nuevos tipos de datos en cantidades inimaginables que tendrán que ser tratados, interpretados y analizados mediante herramientas como el Big Data.

La Internet de las cosas ayudará a ganar eficiencia tanto empresarial como personal, supone un avance con un gran impacto sobre la sociedad y los negocios. Se espera que traiga consigo beneficios como pueden ser: mejorar las experiencias y la satisfacción de los consumidores y usuarios, efectividad de costos, beneficios en la salud, el transporte y la seguridad pública, optimización de la cadena de abastecimiento, entre otros.

El concepto Big Data engloba todos los datos que no pueden ser tratados y analizados con los métodos tradicionales, debido a que se trata en su mayoría de datos no estructurados y que se conservan en una arquitectura distribuida. Hoy en día, enfrentarse al reto que supone el Big Data trae consigo una serie de problemas, ya que supone un desafío para el mundo de las organizaciones, una gran inversión debido a la implementación de una infraestructura tecnológica preparada para este nuevo paradigma, profesionales altamente cualificados y preparados en estas nuevas competencias y un uso correcto de los derechos de privacidad e intimidad de las personas, ya que el uso de la información de los usuarios en este contexto debe estar protegida sin perder el equilibrio entre la libertad de expresión y el derecho a la información. 
Los datos creados en el día a día tienen cada vez más peso e importancia en el buen funcionamiento y desarrollo de organizaciones y empresas. Su uso es cada vez más relevante y clave en los escenarios de evolución de estas instituciones. La capacidad de generación de datos de la sociedad actual en la que vivimos está abrumando a empresas e instituciones. Sobre todo, aquellas que están más enfocadas al cliente, como las empresas de gran consumo, que se ven incapaces de aprovechar esta información.

En el contexto de este nuevo paradigma que es el Big Data los datos son fundamentales para mejorar el rendimiento y comprender mejor el entorno en que se mueven las organizaciones. También ofrece transparencia y mejora el uso de la información beneficiando a muchos sectores, como también ayuda a comprender el uso que hacen los usuarios de la información, ya que a partir de estos datos se pueden saber sus necesidades y demandas. Las instituciones tienen que aprender a gestionar estos datos, tienen que ser capaces de adquirir los mecanismos necesarios para capturar, almacenar, gestionar y analizar este enorme volumen de información.

\section{REFERENCIAS BIBLIOGRÁFICAS}

AIDEN, Erez (2013). Uncharted Big Data as a lens on human culture. New York: Penguin.

ALEXANDRES FERNÁNDEZ, Sadot (2006): "La tecnología de identificación por radiofrecuencia", en Anales de mecánica y electricidad. Asociación de ingenieros del ICAI.

BAUER, Jonathan (2014). "Mobile application for monitoring and controlling devices". Disponible en: www.google.com/patents/US20140245160. [Consulta: 10/10/2014]

Big Data now: 2012 Edition (2012). United States, O'Reilly Media.

BOYD, Dannah (2012). "Critical questions for big data", en Information, Communication \& Society. no. 5, vol. 15, pp.662-679.

BOYD, Dannah; CRAWFORD, Kate (2011). "Six provocation for Big Data". Disponible en: http://papers.ssrn.com/sol3/papers.cfm?abstract_id=1926431. [Consulta: 12/10/2014]

CISCO. Internet será cuatro veces más grande en 2016 (2014). Disponible en: www.cisco.com/web/ES/about/press/2012/2012-05-30-internet-sera-cuatroveces-mas-grande-en-2016--informe-vini-de-cisco.html. [Consulta:10/10/2014]

Clegg, Dai. "Big Data: The Data Velocity Discussion". Disponible en: http://thinking.netezza.com/blog/big-data-data-velocity-discussion. [Consulta: $13 / 10 / 2014]$

CUKIER, Kenneth; MAYER, Viktor (2013). Big Data: La revolución de los datos masivos. Madris: Turner. 
DOUKAS, Charalampos (2012). Building Internet of things with the Arduino. CreateSpace. Disponible en: www.createspace.com/3789612. [Consulta: 14/10/2014]

Empathic things. Intimate computing from wearables to biohacking (2014). Disponible en: www.ict-books.com/books/inspiration-trends/vint-reportthings2-en-detail. [Consulta: 11/10/2014]

ESCOBAR DE LIMA, Carlos Alberto (2007). "Diseño e implementación de un sistema hospitalario basado en tecnología RFID: computación ágil, inteligente y móvil”.

http://revistas.udistrital.edu.co/ojs/index.php/revcie/article/view/292/414.

[Consulta: 11/10/2014]

Decoding Big Data: The corporate race to turn information into profit (2013). United Kingdom: Financial Times.

FUNDACIÓN DE LA INNOVACIÓN BANKINTER (2010). "Cloud Computing: la tercera ola de las tecnologías de la información". Disponible en: www.fundacionbankinter.org/es/publications/cloud-computing. [Consulta: $10 / 10 / 2014$ ]

FUNDACIÓN DE LA INNOVACIÓN BANKINTER (2011). El Internet de las Cosas. Disponible en: www.fundacionbankinter.org/system/documents/8168/ original/XV_FTF_El_internet_de_las_cosas.pdf.[Consulta: 12/10/2014].

GONZÁLEZ, Francisco (2013) "Knowledge Banking for a Hyperconnected Society". Disponible en:www.bbvaopenmind.com/en/book/19-key-essays-onhow-internet-is-changing-our-lives/ [Consulta: 12/10/2014]

GREENFIELD, Adam (2006). Everyware: The Dawning Age of Ubiquitous Computing. United Kingdom: New Riders

GUNGOR, V.C (2013). "A survey on Smart Grid potential applications and communications requirements", en IEEE Transactions on industrial informatics. vol 9, no.1, pp. 28-42.

HANSMANN, Uwe (2003). Pervasive Computing: The Mobile World. Berlin: Springer

HEY, Tony; TANSLEY, Stewart; TOLLE, Kristin (2009). The fourth paradigm: Data-Intensive Scientific Discovery. Redmond: Microsoft Research.

NEEDHAM, Jeffrey (2013). Disruptive possibilities: How Big Data Changes everything. United States: O'Reilly Media.

SCHULZ, Gary (2014). "Radio system for long-range high-speed wireless communication". Disponible en: www.google.com/patents/WO2014124403A1 ?cl=en. [Consulta: 12/10/2014]

FERREYRA RODRÍGUEZ, Carlos Jonathan (2010). "Desarrollo de un sistema de tecnologías de cómputo, comunicaciones y geolocalización". Instituto Politécnico Nacional, Centro de Investigación y de Estudios Avanzados: Departamento de Computación. 
VÁZQUEZ, Juan Ignacio (2014). "The Internet of Things: Outlook and challenges". Disponible en: www.bbvaopenmind.com/en/book/19-key-essayson-how-internet-is-changing-our-lives/. [Consulta: 14/10/2014]

WANT, Roy (2000). "Remembering Mark Weiser : Chief technologist, Xerox PARC", en IEEE Personal Communications, vol.7, no.1, pp. 8-10.

WEISER, Mark (1991). "The Computer for the 21st Century". Disponible en: www.ubiq.com/hypertext/weiser/SciAmDraft3.html. [Consulta: 12/10/2014]

WEISER, Mark (1999). "The origins of ubiquitous computing research at PARC in the late 1980s", en IBM Systems Journal, vol. 38, no 4, pp. 693-696.

WEISER, Mark (1993). "Some computer science issues in ubiquitous computing", en Communications of the ACM - Special issue on computer augmented environments: back to the real world, vol.36, no.7, pp.75-84.

WEISER, Mark (1993). "Ubiquitous computing". Disponible en: www.ubiq.com/ hypertext/weiser/UbiHome.html. [Consulta: 12/10/2014] 